\title{
Stereolithography- A Diagnostic Tool In Oral And Maxillofacial Surgical Treatment Planning
}

\author{
Yashwanth Yadav¹, Pavan Kumar B2, \\ Satish Kumar Desopantula3 ${ }^{3}$ Haripriya Chari ${ }^{4}$
}

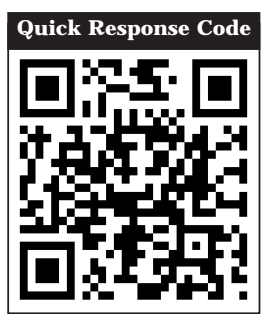

doi: 10.5866/2017.9.10098

${ }^{1 \& 4}$ Senior Lecturer

${ }^{2}$ Professor \& HOD

${ }^{3}$ Post Graduate Student

Department of Oral \& Maxillofacial Surgery,

Kamineni Institute of Dental Sciences,

Narketpally, Nalgonda, Telangana State.

\section{Article Info:}

Received: April 10, 2017

Review Completed: May 11, 2017

Accepted: J une 12, 2017

Available Online: J une, 2017 (www.nacd.in)

(c) NAD, 2017 - All rights reserved

\section{Email for correspondence:}

satishdoc82@gmail.com

\begin{abstract}
:
Stereolithography has recently been introduced as an adjunct to traditional methods of treatment planning for surgical correction of dentofacial deformities. Three dimensional (3D) reformatting of Computer Tomography (CT) images has simplified visualization of maxillofacial deformities, but an accurate 3D model may better support patient education and informed consent andpreoperative analysis of spatial movements of bony structures, fabrication of templates for autogenous grafts, and post-operative analysis. The purpose of this paper is to understand the art and science of stereol ithography and their role in surgical planning in various situations of maxillofacial deformities for the benefit of the patient and surgeon.
\end{abstract}

Key words: Stereolithography, Rapid prototyping, CT imaging, Diagnostic tool.

\section{INTRODUCTION}

The most spectacular technique in the innovation-oriented field of imaging is stereolithography. However there are still limitations such as the precision of details, which is dependent on technical limits (pixel size, slice thickness) and the artifacts of CT scanning, the representation of bone structures without contact with surrounding bone structures, and the cost of stereolithographic models. ${ }^{1}$ These become cost effective only if their application transcends from being merely illustrative to being undeniably valuable in the post operative planning of surgery and eventually the outcome of surgical procedure.

\section{THE BASIC PROCESS ${ }^{2}$}

1. Create a CAD model of the design

2. Convert the CAD model to STL format

3. SlicetheSTL fileinto thin cross sectional layers

4. Construct the model one layer atop another

5. Clean and finish the model 


\section{TECHNIQUE}

The preoperative CT images are obtained (Figure $1 \&$ 2). A Stereolithography RP (Rapid Prototyping Technology) consists of a bath of photosensitive resin, a model building platform and an ultra violet (UV) laser for curing the resin. A mirror is used to guide the laser focus on the surface of the resin where the resin becomes cured when exposed to ultra violet radiation. ${ }^{3-6}$ The mirror is computer controlled its movements being guided to cure resin on a slice by slice basis. A model is initially designed with CAD software in a suitablefile format (Commonly STL) and transferred to the stereolithographic machine for building. The CAD data file is converted in to individual slice of known dimensions.This slice data is the fed to the RP machine, which guides the exposure path of the UV laser on to the resin surface. The layers are cured sequentially and bond together to form a solid object beginning from the bottom of the model and building up. As becomes hardness. After layer of resin is exposed, the resin platform is lowered within the bath by a small known distance. A new layer of resin is wiped across the surface of the previous layer of using a wiper blade. And this second layer is subsequently exposed and cured. The process of curing and lowering platform in to the resin bath is repeated until the full model is complete. The self adhesive property of material causes the layers to bond to one another and eventually form a complete, robust, 3Dobject. The model is then removed from the bath and cured for a further period of time in a UV cabinet. The built part may contain layers which significantly over hang the layers below. If this is the case, then a network of support structures made of the same material is added beneath these overhanging layers at the design state, to add support during the curing process. These support structures analogous to a scaffold are removed by hands after the model is fully cured (Figure 3). This is a labor intensive and time consuming process. Generally, stereol ithography is considered to provide the greatest accuracy and best surface finish of any RP technology. The model material is robust, slightly brittle and relatively light, although it is hygroscopic and may physically warp over time (a few months) is exposed to high humidity. The Osteotomy, Internal Fixation and Augmentation done on Biomodels was shown in Figure 4. The bio models provide a more realistic tool that is easier to handle than computer graphic images. ${ }^{7}$

The purpose of this paper was to assess whether stereolithography technology can assist in surgical treatment planning with regard to following parameters.

1. Preoperative planning and surgical simulation like:
a. Selection of implant i.e., size and shape. ${ }^{4}$
b. Evaluation of bone availability for placement of internal fixation.
c. Measurement of displacement after osteotomy, anticipation of size and form of bone graft.

2. Providing intra operative references during surgery as the model can be sterilized and can be brought into operating room.

3. Decreasing theintra operativetime by allowing the surgeonsto rehearse incisions, measure grafts and fits surgical resections before they operate. $^{5}$

\section{SUMMARY AND CONCLUSION}

Thus MRP (Medical Rapid Prototyping) modeling is undeniably valuable in the practice of oral and maxillofacial surgery. These models serve as indispensable tools for planning of complicatedmaxillofacial procedures such as correction of post traumatic defects, orthognathic surgeries, cleft alveolus and palate and transport distraction of the mandible. This technology is helpful in harvesting quantitative amount of bone graft from donor site, process of adapting the device and typing to fix it was longest and the most tedious part of surgery which also improved. After we started making models, the intra operative time was drastically reduced. The advantage of MRP technology in oral and maxillofacial surgical treatment can be summarized as follows:

1. Easy tolerated by the patient as it is noninvasive procedure.

2. Less expensive compared to models made by other MRP technologies, our method (3D) fabricates models that are relatively inexpensive, dimensionally accurate and reasonably strong and workable.

3. Quick to perform, less time consuming. The models can be fabricated in 1 to 2 days after CT scan is completed.

4. The post operative outcome of procedure is certainly better. Thus the models improve the prognosis. 


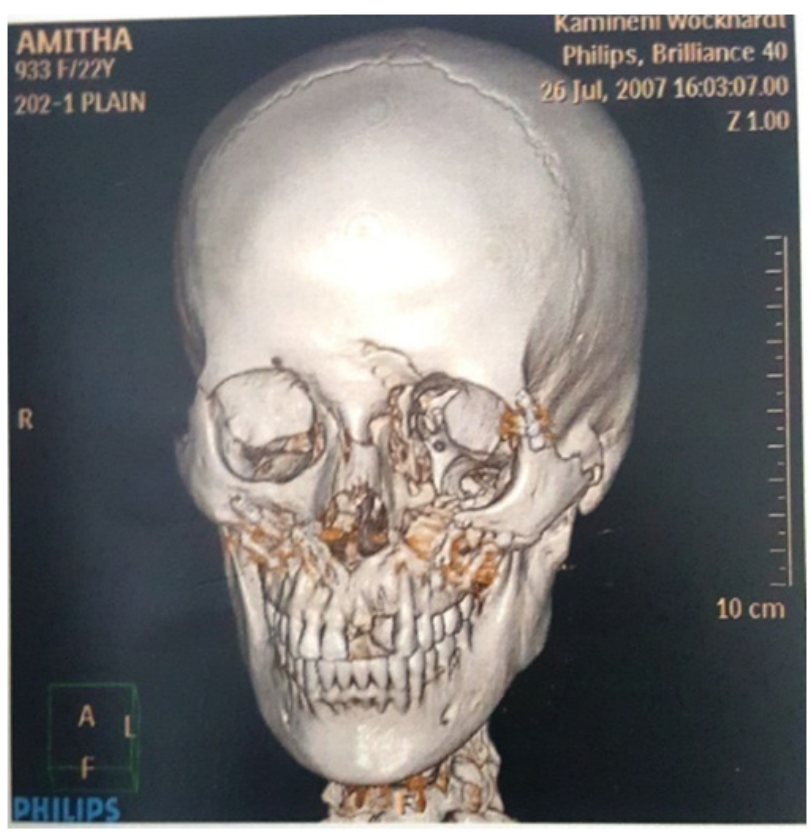

Figure 1: 3D CT Image of skull showing frontal view

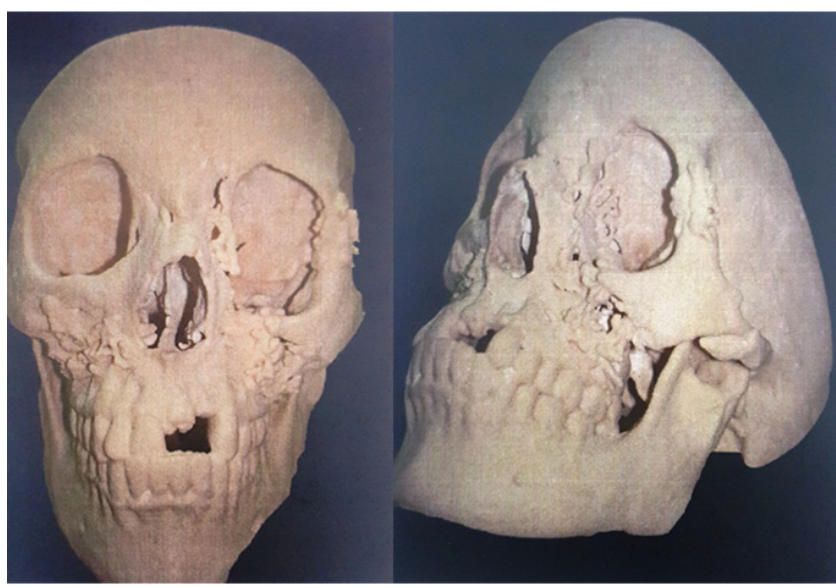

Figure 3: Stereolithographic model showing residual deformity

5. Helps the surgeon to clearly visualize the osteotomy cuts for repositioning and alignment of bony fragments to their correct anatomical position.

\section{REFERENCES}

1. Winder J, Bibb R. Medical rapid prototyping technologies: State of the art and current limitation for application in oral and maxillofacial surgery. J Med Eng Technol 1999; 23(1):26-8.

2. Ferry PW, Melchels, J an Feijen. A review on stereolithography and its applications in biomedical engeneering. Biomaterials 2010; 31:6130.

3. Mole C, Gerard H. A new three dimensional treatment

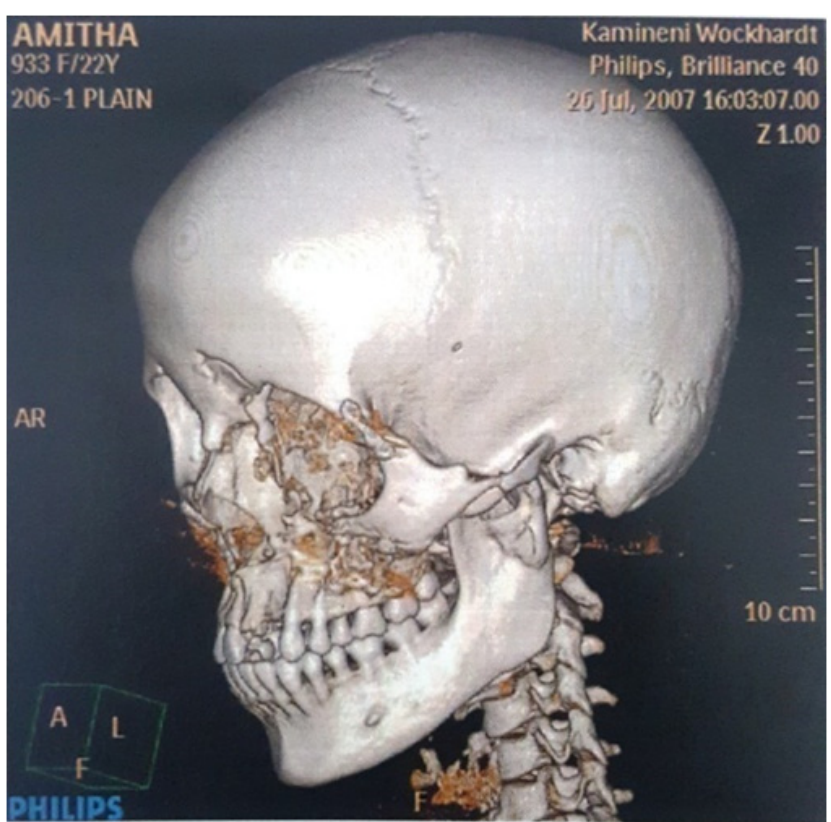

Figure 2: 3D CT I mage of skull showing lateral view

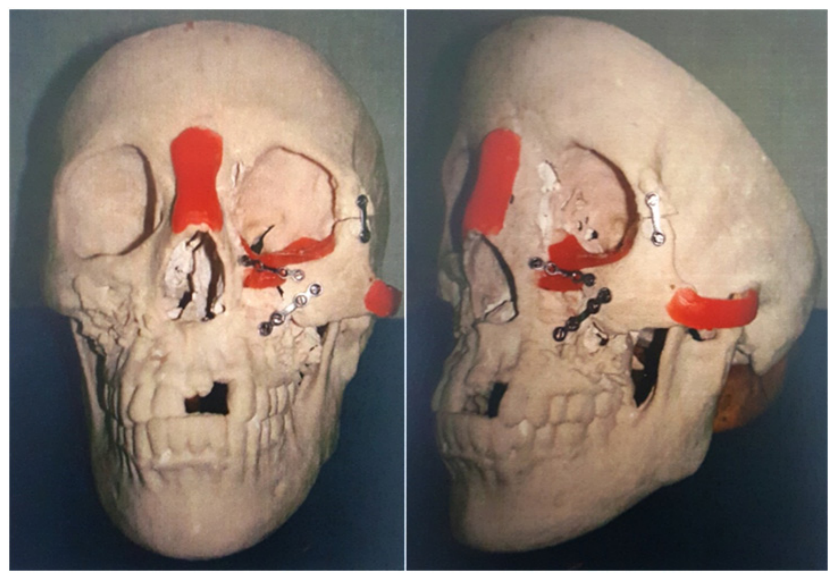

Figure 4: Bio-model showing the osteotomy, internal fixation and augmentation.

algorithm for complex surfaces: Applications in Surgery. J Oral Maxillofac Surg 1995; 53(2):158-62.

4. Sarment DP, Sukovic P. Accuracy of implant placement with a stereolithographic surgical guide. Int J Oral Maxillofac I mplants 2003; 8(4):332-6.

5. GabrieleTonellini, Fabio Mazzoleni. Virtual surgery simulation in orbital wall reconstruction: Integration of surgical navigation and stereolithographic models. J Cranio-Maxillofacial Surg 2014; 42(8):2025-34.

6. Bill J S, Ruther JF. Stereolithography in Oral and maxillofacial operation planning. Int J Oral Maxfac Surg 1995; 24(1):98-103.

7. Abe M, Tabuchi K, Goto M. A model based surgical planning and simulation of cranial base surgery. Neurol Med Chir (Tokyo) 1998; 38(11):746-50. 